\title{
Wie kann günstiger Wohnraum definiert werden und was sind sinnvolle Maßnahmen, um diesen zu erweitern?
}

\author{
Johann Bacher* und Dennis Tamesberger ${ }^{* *}$
}

\section{Zusammenfassung}

Der vorliegende Beitrag untersucht, wie günstiger Wohnraum definiert werden kann, wie hoch die Anzahl an günstigen Mietwohnungen in Österreich ist und welche Einflussfaktoren es gibt. Durchgeführt wird eine Sekundärdatenanalyse des Mikrozensus des Jahres 2017. Als günstig wurden dabei in Anlehnung an das Konzept des Kantons Luzern Wohnungen bezeichnet, deren Hauptmiete weniger als $70 \%$ der Durchschnittsmiete in Österreich beträgt. In Bezug auf die ausgewählten Haushalte mit einem Nettoeinkommen knapp über der Einkommensgrenze der Wohnbeihilfe resultieren Mietkostenbelastungen von $21 \%$ bis $25,7 \%$. Von einem sozialpolitischen Standpunkt aus betrachtet stellt die $70 \%$-Schwelle eine sehr ambitionierte Zielsetzung dar. In Österreich erfüllen 398.000 Hauptmietwohnungen bzw. 24 \% aller Hauptmietwohnungen dieses Kriterium. Allerdings wurden von diesen Wohnungen nur 70.400 in den letzten drei Jahren vermietet. Als wichtige Strategie erscheint daher nicht nur mehr Wohnungen zu bauen, sondern vor allem den Anteil an günstigen Hauptmietwohnungen bei Neubauten bzw. Neuvermietungen zu erhöhen.

Schlagwörter: leistbares Wohnen, günstiges Wohnen, Mikrozensus, Mietpreise, Sozialpolitik

\section{Availability and use of affordable housing in Austria \\ How can cheap housing be defined and what are reasonable measures to expand it?}

\begin{abstract}
This paper examines how affordable housing can be defined, what the total number of cheap rented apartments in Austria is and which influencing factors there are. For this purpose, a secondary data analysis of the 2017 micro-census is being carried out. In accordance to the concept of the Swiss canton of Lucerne, apartments are being referred to as cheap if their headlease is $70 \%$ lower than the average rent in Austria. The rent burden of the selected households with a net income just over the income limit of the housing benefit results between 21 $\%$ and $25.7 \%$. From a socio-political point of view, the 70 percent threshold represents a very ambitious target. 398.0oo apartments that are rented for headlease in Austria - corresponding to $24 \%$ of these apartments - fulfil this criterion. However, only 70.400 of these apartments have been leased in the past three years. An important strategy seems hence not only to build apartments, but above all to increase the proportion of apartments that are rented for headlease when it comes to new buildings or new lettings.
\end{abstract}

Keywords: affordable housing, cheap housing, micro-census, rents, social policy, Austria

\footnotetext{
${ }^{*}$ Johann Bacher, Institut für Soziologie, Johannes Kepler Universität Linz. E-Mail: johann.bacher@jku.at.

** Dennis Tamesberger, Abteilung für Wirtschafts-, Sozial- und Gesellschaftspolitik der Arbeiterkammer Oberösterreich. E-Mail: tamesberger.d@akooe.at

Wir danken der AK OÖ für die finanzielle Unterstützung dieser Forschungsarbeit und einer/einem der anonymen BegutachterInnen, die/der sowohl durch engagierte methodische als auch inhaltliche Kommentare zur Weiterentwicklung des Artikels beigetragen hat.
} 


\section{Einleitung}

Wohnen ist ein menschliches Grundbedürfnis. Gleichzeitig bestimmt die soziale Lage den Zugang zu Wohnraum und die Qualität der Wohnverhältnisse. Wer wenig Geld hat, wohnt häufig in kleineren Wohnungen mit schlechteren Ausstattungskategorien, mit weniger Zugangsmöglichkeiten zu Natur und Naherholung, aber auch mit weniger qualitativen sozialen Betreuungs- und Bildungsangeboten in der Nähe des Wohnortes. Damit bestimmt die Wohnungslage auch die sozialen Verhältnisse. Hinzu kommt, dass ärmere Bevölkerungsgruppen von der Wohnkostenbelastung im Verhältnis zu ihrem verfügbaren Einkommen stärker betroffen sind und somit Wohnungspreise bzw. Mietpreise soziale Ungleichheit beeinflussen (Holm 2018). Auf der anderen Seite sind Immobilienpreise eng verknüpft mit Vermögens- und Verschuldungsstrukturen von Haushalten (Springler 2014), womit die Wohnungsfrage als fundamentale Verteilungsfrage erscheint.

$\mathrm{Zu}$ Recht erhält das Thema des leistbaren Wohnens auch im öffentlichen Diskurs in Österreich vermehrt Aufmerksamkeit. ${ }^{1}$ In der Forschung bekommt das Thema Wohnen ebenfalls erhöhte Aufmerksamkeit (siehe u. a. Lang und Stöger 2018; Beer und Wagner 2017; Kunnert 2016; Mundt 2017; OeNB 2018; Schoibl und Stöger 2014; Statistik Austria 2018; Stöger 2017; Tockner 2017; Zucha 2016). So haben das Wirtschaftsforschungsinstitut (WIFO) (Kunnert 2016), die Oesterreichische Nationalbank (OeNB) (Beer und Wagner 2017) und das Institut für Immobilien, Bauen und Wohnen $\mathrm{GmbH}$ (IIBW) (Mundt und Amann 2015; Mundt 2017) Studien zum leistbaren Wohnen vorgelegt. Die Mietpreisentwicklung in Wien wurde von Tockner (2017) untersucht. Trotz dieser Aufmerksamkeit scheint - zumindest für Österreich - ungeklärt zu sein, wie günstiger, leistbarer Wohnraum valide und plausibel definiert werden kann. Ein praxistauglicher Indikator könnte die Basis für ein systematisches und regelmäßiges Monitoring des Angebots an günstigem Wohnraum in Österreich liefern, wie es etwa andere Indikatoren im Bildungsbereich oder in der Arbeitsmarkt- und Armutsforschung leisten. Politisch könnten Ziele für den Bedarf oder für den Ausbau an günstigem Wohnraum festgelegt, Maßnahmen zur Erreichung der

1 Der ORF Wien reihte das Thema unter die Top Fünf der wichtigsten Themen in Österreich. Online: http:// wien.orf.at/news/stories/2869309/ [29.01.2019].
Ziele gesetzt und ihre Effektivität regelmäßig überprüft werden.

Dieser Beitrag soll hierfür eine Grundlage bieten. Auf der Basis eines neu entwickelten Indikators für Österreich, der die Verfügbarkeit und Nutzung von günstigem Wohnraum misst, werden folgende Fragestellungen behandelt:

1. Wie lässt sich günstiger Wohnraum definieren?

2. Wie hoch sind Anteil und Anzahl günstiger Mietwohnungen in Österreich?

3. Von welchen Faktoren hängt das Angebot an günstigen Mietwohnungen $\mathrm{ab}$ ?

Die Forschungsfragen sollen durch eine Sekundärdatenanalyse der Mikrozensuserhebung des Jahres 2017 beantwortet werden. Der vorliegende Beitrag ist wie folgt gegliedert. Kapitel 2 bietet einen Überblick über den aktuellen Forschungsstand zu Einflussfaktoren auf Miet- und Wohnungspreise. Kapitel 3 beschreibt die Datengrundlage und das methodische Vorgehen. Anschließend werden die Ergebnisse präsentiert (Kapitel 4). In einer zusammenführenden Diskussion werden die Qualität des entwickelten Indikators und dessen Grenzen angeführt sowie Maßnahmenvorschläge für Österreich skizziert (Kapitel 5).

\section{Einflussfaktoren auf Miet- und Wohnungspreise}

Der aktuelle Forschungsstand verweist sowohl auf angebots- als auch nachfrageseitige Einflussfaktoren auf Mietpreise. Angebotsseitig wird der Mietpreis insbesondere durch den Wohnungsbestand und jene Faktoren, die diesen beeinflussen, bestimmt. Übersteigt das Angebot an Mietwohnungen die Nachfrage, werden die Mietpreise sinken und vice versa. Weiters liegt ein Zusammenhang zwischen der Qualität der Wohnungen und den Mietpreisen nahe. Demnach ist anzunehmen, dass Mietpreise mit dem Alter der Wohnungen abnehmen (Fritzsche und Kluge 2014). Blaas und Wieser (2004) analysierten die Entwicklung der Wohnungsmieten in den 1980er- und 1990er-Jahren auf Bundesländerebene in Österreich und ermittelten als Haupteinflussfaktor die Anzahl der gefördert errichteten Wohnungen, die den Mietpreis reduzieren. Ihrer Schätzung zufolge bedeutet beispielsweise eine Senkung der Neubauförderung um 10 \% kurzfristig einen Anstieg der realen marktorientierten Mieten um rund $1 \%$. Ebenfalls erklärt die Statistik Austria regionale Unterschiede bei den Mietpreisen mit dem unterschiedlichen Anteil an Gemeinde- und Genossenschaftswohnungen (Zucha 2016). Ähnliche 
Befunde werden für Deutschland berichtet (Fehlberg und Mießner 2015; Institut für Wohnen und Umwelt (IWU) 2005). Sozialer Wohnbau wirkt senkend auf die Mietpreise, weil günstiger Wohnraum geschaffen wird (Angebotseffekt). Gleichzeitig bestehen aber auch wechselseitige Effekte zwischen öffentlichem und privatem Wohnungsmarkt. Bei einem geringen Anteil an sozialem Wohnbau sind vermehrt Menschen auf den privaten Wohnungssektor angewiesen und durch die erhöhte Nachfrage steigen die Mietpreise (Nachfrageeffekt) (Egner und Grabietz 2017).

Darüber hinaus finden Blaas und Wieser (2004) Hinweise dafür, dass Änderungen im Mietrecht in den 1990er-Jahren in Österreich $\mathrm{zu}$ einem höheren Mietniveau geführt haben, da zum einem nach der Mietrechtsänderung ein geringerer Anteil der Mietwohnungen einer Mietregulierung unterlag und zum anderen Mieterhöhungen im Bestand erleichtert wurden. Eine Mikrozensus-Analyse für Wien (Tockner 2017) verweist auf eine weitere Problematik, dass nämlich befristet vermietete Wohnungen teurer sind als unbefristete. Es wird davon ausgegangen, dass bei befristeten Verträgen, die nach einer bestimmten Zeit neu abgeschlossen und verhandelt werden, es zu ungerechtfertigten Zuschlägen kommt.

In Hinblick auf die Nachfrage spielt die demografische und wirtschaftliche Entwicklung eine Rolle, wobei diese auch angebotsseitig wirken kann. Die demografische Entwicklung kann Mietpreise theoretisch in mehrerlei Hinsicht beeinflussen. Anzunehmen ist, dass entlang des Lebenszyklus Menschen unterschiedliche Wohnbedürfnisse haben und somit entsprechend dem Alter unterschiedliche Nachfrageeffekte auslösen, womit der Mietpreis beeinflusst wird (Fritzsche und Kluge 2014). Girouard et al. (2006) sprechen z. B. von einem "household formation age“, wonach jenes Alter, in dem häufig ein eigener Haushalt gegründet wird oder dieser aufgrund von Kindern bzw. Lebensgemeinschaften erweitert wird, die Nachfrage nach Wohnungen und somit den Mietpreis erhöht. Anders ausgedrückt bedeutet dies, dass ein höherer Bevölkerungsanteil in einer Region im sogenannten „household formation age“ den Mietpreis erhöhen kann. Während Fritzsche und Kluge (2014) das „household formation age“ der Altersgruppe zwischen 30 und 49 Jahre zuordnen, zeigen Egner und Grabrietz (2017), dass auch der Anteil der Studierenden einen erhöhenden Einfluss auf den Mietpreis hat. Auf der anderen Seite kann auch der Anteil von älteren Menschen in einer Region Auswirkungen auf den Mietpreis haben. Ältere Menschen sind tendenziell weniger mobil und bleiben häufig in der bestehenden Wohnung, selbst wenn die aktuelle Wohnungsgröße aufgrund des Auszuges der Kinder und/oder eines eventuellen Todes des Lebenspartners nicht mehr notwendig wäre. Durch dieses Beharrungsverhalten entsteht ein Angebotseffekt. Die Zahl des Wohnungsangebots an größerem Wohnraum bleibt ceteris paribus gleich und führt bei steigender Nachfrage durch die nachkommenden Generationen $\mathrm{zu}$ einem Druck auf die Mietpreise für größeren Wohnraum.

Ein weiterer nachfrageseitiger Einflussfaktor ist das Bevölkerungswachstum (Wieser o. J.) und hier insbesondere die Zuwanderung. Höhere Zuwanderung in einer bestimmten Region erhöht die Wohnungsnachfrage und lässt somit höhere Mieten erwarten (Fritzsche und Kluge 2014). Empirische Hinweise hierfür haben beispielsweise Fritzsche und Kluge (2014) für Deutschland oder Saiz (2003; Saiz 2007) für amerikanische Städte gefunden. Bei der Analyse der Wohnungsmietpreise im Ruhrgebiet hatte hingegen der AusländerInnenanteil kaum eine Erklärungskraft (Möbert et al. 2008).

Die wirtschaftliche Entwicklung hat insofern eine Bedeutung für die Mietpreise, da bei hoher Arbeitslosigkeit mehr (günstige) Mietwohnungen als Eigentumswohnungen nachgefragt werden. Dies kann zu dem paradoxen Effekt führen, dass Mietpreise im unteren Preissegment wegen der größeren Nachfrage steigen, da der Einkommensverlust durch die Arbeitslosigkeit eher dazu führt, dass nicht in den Erwerb von Wohnungseigentum investiert werden kann (Blaas und Wieser 2004), sodass mehr Mietwohnungen nachgefragt werden. Wurde bereits Wohnungseigentum angeschafft und wurden dafür Kredite aufgenommen, kann die Situation auftreten, dass dieses verkauft und eine Mietwohnung gesucht werden muss.

Wohnungsmärkte weisen jedoch einige Besonderheiten auf, die sie von einem Standardmarktmodell unterscheiden. Das Wohnungsangebot ist grundsätzlich regional und eine Änderung des Wohnungsangebots kann nicht unmittelbar erfolgen, sondern setzt aufgrund von Planungs- und Bauphasen zeitverzögert ein. Das Flächenangebot ist langfristig zumindest in Großstädten oder auch aufgrund von geografischen Bedingungen (z. B. wegen Flüssen oder Bergen) beschränkt. Darüber hinaus erscheint der Wohnungsmarkt zumindest in zwei Teilbereiche segmentiert $\mathrm{zu}$ sein. Erstens in jenen mit älteren bestehenden Wohnungen, für die vertraglich vereinbarte Bestands- 
mieten gezahlt werden. Zweitens in ein Wohnungssegment, in dem deutlich höhere Neuvertragsmieten verlangt werden. Die Preise am Wohnungsmarkt sind relativ intransparent, da die meisten Transaktionen und Verträge bilateral abgeschlossen werden. Mieten sind auch keine flexiblen Preise, da sie vertraglich festgelegt bzw. durch Mieterschutz reguliert sind. Eine Nachfrageänderung als Reaktion auf ein verändertes Wohnungsangebot wird aufgrund von längerfristigen Mietverträgen daher ebenfalls zeitverzögert stattfinden. Hinzu kommt, dass die Transaktionskosten (z. B. für Übersiedlungen) und die Anteile an externer Finanzierung relativ hoch sind, was Marktreaktion verzögert (Priewe 2019; Schönig 2013; Springler 2014; Wieser o.J.). Zu beachten ist schließlich auch, dass die Preisbildung auf Wohnungsmärkten von Finanzmärkten und ihren Zinsen abhängt, die die Vermögens- und Verschuldungsentwicklungen beeinflussen (Springler 2010; Springler 2014; Wieser o. J.)

Ausgehend vom aktuellen Forschungsstand wird versucht, die Einflussfaktoren zu operationalisieren, und untersucht, welchen Einfluss die genannten Merkmale auf die Wahrscheinlichkeit haben, dass eine Wohnung in Österreich günstig vermietet ist.

\section{Datenbasis und methodisches Vorgehen}

\subsection{Daten}

Die Forschungsfragen sollen durch eine Sekundärdatenanalyse der Mikrozensuserhebung des Jahres 2017 beantwortet werden. Im Mikrozensus (MZ) wird pro Quartal eine Stichprobe von rund 22.500 Haushalten befragt, in denen etwa 45.00o Personen leben (Statistik Austria 2018e). Dabei handelt es sich um eine Stichprobe mit Fünftelrotation, d. h. quartalsweise beendet ein Fünftel der Haushalte den Befragungszyklus und ein Fünftel neuer Haushalte beginnt damit. Diese Methode ermöglicht einerseits das Messen von Veränderungen, und andererseits werden mit der quartalsweisen Erneuerung der Stichprobe auch Veränderungen in der Grundgesamtheit erfasst.

Zur Ermittlung von günstigen Wohnungen werden die Mieten inklusive Betriebskosten, aber ohne Energiekosten herangezogen. Zur Berechnung der Zahl der günstigen Wohnungen werden nur Hauptmietwohnungen in Betracht gezogen. Bei allen anderen Rechtsformen wird angenommen, dass sie als günstige Wohnungen nicht dem Wohnungsmarkt als Mietwohnungen zur Verfügung stehen. Am gesamten
Wohnungsbestand haben Hauptmietwohnungen einen Anteil von 42,1 \%. In der Prozentuierungsbasis der ca. 3,9 Millionen Wohnungen (inkl. Eigenheimen) nicht enthalten sind Leerstände von Wohnungen.

\subsection{Operationalisierung von günstigem Wohnraum}

Zur Messung der Verfügbarkeit von günstigem Wohnraum folgen wir dem Konzept des Kantons Luzern (LUSTAT Statistik Luzern 2018). Als preisgünstig gelten Wohnungen, deren Mietpreis weniger als $70 \%$ des durchschnittlichen Mietpreises der Wohnungen mit gleicher Zimmeranzahl beträgt. Darauf aufbauend bilden wir einen Index (GÜNSTIG7o), der annimmt, dass eine günstige Mietwohnung $i$ mit $k$ Zimmern vorliegt, wenn die Miete inkl. Betriebskosten weniger als $70 \%$ der durchschnittlichen Miete inkl. Betriebskosten aller Mietwohnungen mit $k$ Zimmern beträgt:

$$
\mathrm{x}_{\mathrm{i \varepsilon k}}<0,7 \cdot \overline{\mathrm{x}}_{\mathrm{k}^{\prime}} \text {, wobei }
$$

$\overline{\mathrm{x}}_{\mathrm{k}}$ die durchschnittliche Miete inklusive Betriebskosten von Mietwohnungen mit $k$ Räumen ist. $\mathrm{x}_{\mathrm{i} k \mathrm{k}}$ sind die Mietkosten (Miete inkl. Betriebskosten) einer Wohnung $i$ mit $k$ Räumen. Der Index GÜNSTIG7o erfasst alle Mietwohnungen unabhängig davon, wann das Mietverhältnis eingegangen wurde. Er misst somit den Gesamtbestand an günstigen Wohnungen und bildet rezente Entwicklungen, die für die Politik von besonderem Interesse sind, nicht adäquat $a b .^{2}$ Daher wird ein zweiter Indikator (GÜNSTIG703) verwendet, der die Dauer des Mietverhältnisses berücksichtigt. Er misst, wie viele günstige Wohnungen in den letzten drei Jahren vermietet wurden, und ist definiert als

$$
\mathrm{x}_{\mathrm{it \varepsilon k}}<0,7 \cdot \overline{\mathrm{x}}_{\mathrm{k}} \text { und } \mathrm{t} \leq 3 \text { Jahre. }
$$

Daraus ergeben sich folgende Kenngrößen und Schwellenwerte für günstigen Wohnraum in Österreich (Tabelle 1). Bei beiden Indikatoren handelt es sich um relative Indikatoren. Steigen die Durchschnittsmieten, dann erhöht sich auch der Schwellenwert der günstigen Mietwohnungen, was insbesondere dann problematisch werden kann, wenn die Mieten deutlich schneller wachsen als die Einkommen, wie dies in den letzten

2 Siehe dazu auch das Thema Segmentierung als zentrales Merkmal des Wohnungsmarktes im Literaturüberblick (Kapitel 2). 
Tabelle 1: Kenngrößen und Schwellenwerte für günstigen Wohnraum nach Anzahl der Räume

\begin{tabular}{|c|c|c|c|c|}
\hline $\begin{array}{l}\text { Anzahl der Räu- } \\
\left.\text { me inkl. Küche }{ }^{a}\right)\end{array}$ & $\begin{array}{c}\text { Durchschnittliche } \\
\left.\text { Hauptmiete inkl. } B K^{b}\right)\end{array}$ & $\begin{array}{l}\text { Schwellenwert für gün- } \\
\text { stige Wohnung inkl. } B K^{c} \text { ) }\end{array}$ & $\begin{array}{c}\text { Durchschnittliche } \\
\text { Wohnungsgröße in } \mathbf{m}^{2}\end{array}$ & $\begin{array}{c}\text { Miete pro } m^{2} \text { von gün- } \\
\text { stigen Wohnungen }\end{array}$ \\
\hline 1 & 335,46 & 234,82 & 35 & 6,06 \\
\hline 2 & 422,72 & 295,90 & 50 & 5,65 \\
\hline 3 & 484,86 & 339,40 & 65 & 4,94 \\
\hline 4 & 555,56 & 388,89 & 80 & 4,57 \\
\hline 5 & 688,47 & 481,93 & 101 & 4,29 \\
\hline 6 und mehr & 934,82 & 654,38 & 144 & 4,10 \\
\hline Insgesamt & 505,89 & 354,01 & 68 & 5,01 \\
\hline
\end{tabular}

Quelle: MZ2017, eigene Berechnungen

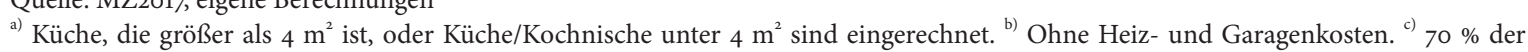
durchschnittlichen Hauptmiete inklusive Betriebskosten

Lesehilfe: Im Jahr 2017 beträgt die durchschnittliche Hauptmiete inkl. Betriebskosten für eine 1-Zimmer-Wohnung € 335,46. Bei Verwendung der $70 \%$-Schwelle ergibt sich eine Mietobergrenze von $€$ 234,82. Die durchschnittliche Wohngröße der 1-Zimmer-Wohnung liegt bei $35 \mathrm{~m}^{2}$. Der maximale Mietpreis pro $\mathrm{m}^{2}$ für eine günstige Wohnung beträgt $€ 6,06$. Er wurde als Durchschnitt der Quadratmeter-Mieten in den günstigen Wohnungen berechnet und weicht daher von der Schätzung ab, wenn der Schwellenwert von $€ 234,82$ mit $_{35} \mathrm{~m}^{2}$ dividiert wird.

Jahren der Fall war. So z. B. stieg die durchschnittliche Hauptmiete inklusive Betriebskosten zwischen 2011 und 2016 um 16,4 \% (Statistik Austria 2018a), während die Nettojahreseinkommen aus unselbstständiger Erwerbstätigkeit in demselben Zeitraum nur um 10,6 \% zunahmen (Statistik Austria 2018c).

Der Skalierungsfaktor von 70 \% wird in LUSTAT Statistik Luzern (2018) nicht näher begründet. Denkbar wäre auch die Wahl eines anderen Skalierungsfaktors $p$ zur Ermittlung des Schwellenwertes $\mathrm{s}_{\mathrm{k} / \mathrm{p}}=\mathrm{p} \cdot \overline{\mathrm{x}}_{\mathrm{k}}$. Deschermeier et al. (2015) beispielsweise setzen in ihrer Studie über Anbieter von günstigem Wohnraum in zehn Großstädten in Nordrhein-Westfalen den Skalierungsfaktor gleich $100 \%$ und wählen als Schwellenwert $\mathrm{S}_{\mathrm{k}}=1,0 \cdot \overline{\mathrm{X}}_{\mathrm{k}}$. Auf der anderen Seite könnte auch überlegt werden, den in der Armutsforschung üblichen Skalierungsfaktor von $60 \%$ zu verwenden.

Zur inhaltlichen Verdeutlichung der Schwellenwerte wurden in Tabelle 2 die Mietkostenbelastungen für unterschiedliche Haushaltszusammensetzungen berechnet, wobei angenommen wird, dass die Haushaltseinkommen knapp über den Einkommensobergrenzen der oberösterreichischen Wohnbeihilfe liegen (Land OÖ 2018b), sodass eine eventuelle Wohnbeihilfe nicht berücksichtigt werden muss. ${ }^{3}$ Als Referenzwert

3 Zu beachten ist aber, dass die oberösterreichische Wohnbeihilfe (Land OÖ 2018a, 2018b) eine Einkommensuntergrenze vorsieht. Wird diese unterschritten, ändert sich die Wohnbeihilfe nicht, sodass in sehr einkommensschwachen Haushalten höhere Mietkostenbelastungen auftreten können, sofern keine weiteren Hilfen bezogen werden. für eine zumutbare Mietkostenbelastung wird dabei ein Schwellenwert von 25 \% verwendet (Statistik Austria 2018d). Entsprechend diesem Schwellenwert wird von einer hohen Mietkostenbelastung dann gesprochen, wenn der Anteil der Mietkosten am Haushaltseinkommen $25 \%$ übersteigt. ${ }^{4}$ Bezogen auf die österreichischen Armutsschwellen (Statistik Austria 2019) ergeben sich die berechneten Belastungsquoten in Haushalten, deren Einkommen $10 \%$ bis $15 \%$ unter der Armutsschwelle liegen.

Entsprechend den Modellrechnungen ergeben sich für einen 1-Personen-Haushalt mit einem verfügbaren monatlichen Nettohaushaltseinkommen von $€ 1.150$ in einer günstigen 2-Zimmer-Wohnung Mietkosten von $€$ 295,90. Dies entspricht einer Mietkostenbelastung von 25,7 \%. Für die anderen untersuchten Haushaltskonstellationen liegt die Mietkostenbelastung bei ca. $21 \%$. Für eine Familie mit zwei Kindern, die in einer günstigen 5-Zimmer-Wohnung lebt, ergibt sich bei einem verfügbaren monatlichen Haushaltseinkommen von $€$ 2.200 eine Mietkostenbelastung von 21,9\%. Würden die Haushalte dagegen die durchschnittliche Miete bezahlen, würden die Belastungen deutlich über $25 \%$ liegen. Für die Familie mit zwei Kindern wären dies 31,3\%.

Bei den hier verwendeten Maßzahlen handelt es sich um relative Indikatoren. Bezugsgröße ist die durchschnittliche Bruttomiete (Nettomiete inkl. BK). Die Zahl der günstigen Wohnungen wird einmal ohne

4 Auf europäischer Ebene wird ein Schwellenwert von $40 \%$ eingesetzt (Statistik Austria 2018d), dieser schließt aber die Energiekosten mit ein. 
Tabelle 2: Kenngrößen und Schwellenwerte für günstigen Wohnraum nach Anzahl der Räume

\begin{tabular}{|c|c|c|c|}
\hline $\begin{array}{c}\text { Haushalts- } \\
\text { Zusammensetzung }\end{array}$ & $\begin{array}{c}\text { Verfügbares monatliches } \\
\text { Haushaltseinkommen }{ }^{a} \text { ) }\end{array}$ & $\begin{array}{c}\text { Miete für leistbare Woh- } \\
\left.\text { nung inkl. } B K^{b}\right) \text { bzw. bei } \\
\text { Durchschnittsmiete }\end{array}$ & $\begin{array}{l}\text { Mietkosten- } \\
\text { belastung in \% }\end{array}$ \\
\hline 1 Person (z. B. alleinstehende Pensionistin); & 1.150 & $295,90^{c)}$ & $25,7 \%$ \\
\hline 2-Zimmer-Wohnung & $(9 \%)^{e)}$ & $422,72^{\mathrm{d})}$ & $36,8 \%$ \\
\hline 2 Erwachsene; & 1.600 & $339,40^{c)}$ & $21,2 \%$ \\
\hline 3-Zimmer-Wohnung & $(15 \%)^{e)}$ & $484,86^{\text {d) }}$ & $30,3 \%$ \\
\hline Alleinerziehende mit 2 Kindern; & 1.850 & $388,89^{c}$ & $21,0 \%$ \\
\hline 4-Zimmer-Wohnung & $(8 \%)^{\mathrm{e})}$ & $555,56^{\text {d) }}$ & $30,0 \%$ \\
\hline Eltern mit 2 Kindern; & 2.200 & $481,93^{c)}$ & $21,9 \%$ \\
\hline 5-Zimmer-Wohnung & $(17 \%)^{\mathrm{e})}$ & $688,47^{d)}$ & $31,3 \%$ \\
\hline
\end{tabular}

Quelle: MZ2017, eigene Berechnungen

a) Der Wert wurde so gewählt, dass in Oberösterreich keine Wohnbeihilfe mehr ausbezahlt wird, sodass das Argument, die tatsächliche Mietkostenbelastung sei geringer, da Wohnbeihilfe bezogen werden kann, nicht greift. ${ }^{\text {b) }}$ Entsprechend den Werten der Tabelle $2 .{ }^{c)}$ Miete für leistbare Wohnung. ${ }^{\text {d) }}$ Durchschnittliche Miete. ${ }^{\text {e) }}$ Prozentwert, mit dem das ausgewiesene monatliche Haushaltseinkommen unter der Armutsschwelle liegt. $9 \%$ bedeutet also, dass der Wert von $€ 1.1509 \%$ unter der Armutsschwelle für einen 1-Personen-Haushalt liegt (Statistik Austria 2019).

Lesehilfe: Lebt eine alleinstehende Person mit einem monatlichen verfügbaren Haushaltseinkommen (Nettoeinkommen) in einer leistbaren 2-Zimmer-Wohnung, dann muss sie 25,7 \% ihres verfügbaren Einkommens für Miete aufwenden. Die tatsächliche Mietkostenbelastung kann geringer sein, da die tatsächliche Miete unter dem Schwellenwert liegen kann. Müsste sie dagegen die durchschnittliche Miete bezahlen, würde sie $36,8 \%$ des verfügbaren Haushaltseinkommens aufwenden.

weitere Bedingung berechnet und einmal mit der zusätzlichen Vorgabe, dass nur Neuvermietungen in den letzten drei Jahren betrachtet werden.

Ein alternativer Zugang wäre die Festlegung eines absoluten Schwellenwertes je $\mathrm{m}^{2}$. Entsprechend einem Modellprojekt ${ }^{5}$ in Tirol (Amt der Tiroler Landesregierung 2017) könnte ein Schwellenwert von $€ 5$ je $\mathrm{m}^{2}$ festgelegt werden. Die oberösterreichische Wohnbeihilfe würde einen Schwellenwert von $€ 7$ nahelegen, da bis zu diesem Betrag Wohnbeihilfe bezogen werden kann.

Um der Sensibilität der Indikatoren im univariaten Bereich Rechnung zu tragen, werden die univariaten Schätzungen für die Zahl der günstigen Wohnungen für unterschiedliche Schwellenwerte vorgenommen. Neben der $70 \%$-Schwelle werden zum einen die $60 \%$-, $75 \%$ - und $80 \%$-Schwelle untersucht und zum anderen eine 5-Euro-, 6-Euro- und 7-Euro-Schwelle. Die sich daraus ergebenden Mietkostenbelastungen sind in Abbildung 1 wiedergegeben. Für eine alleinstehende Pensionistin in einer 2-Zimmer-Wohnung würde sich eine Mietkostenbelastung von 36,8\% ergeben, wenn sie

5 Mit dem 5-Euro-Wohnmodell des Landes Tirol sollen Kleinwohnanlagen mit 15 bis 25 Wohnungen mit zwei oder drei Zimmern gefördert werden. Das angestrebte Entgelt von $€ 5$,0o pro $\mathrm{m}^{2}$ soll die Betriebskosten und Heizkosten beinhalten. Es soll durch eine einfache Ausstattung, reduzierte Außenanlagen sowie den Verzicht auf ein Kellergeschoß und eine Tiefgarage erreicht werden. die Durchschnittsmiete für eine 2-Zimmer-Wohnung zahlen müsste. Wenn sie bei Verwendung der 60 \%-Schwelle in einer günstigen Wohnung leben würde, hätte sie eine Mietkostenbelastung von 22,1\%, die unter der nationalen Schwelle von $25 \%$ (siehe oben) liegen würde. Bei Verwendung der 70 \%-Schwelle wäre, wie bereits dargestellt, die Mietkostenbelastung 25,7\%. Sie würde auf 27,6 \% bzw. 29,4 \% steigen, wenn zur Definition von günstigen Wohnungen die $75 \%$-Schwelle bzw. $80 \%$-Schwelle eingesetzt wird. Bei Verwendung von absoluten Schwellen würde die Mietkostenbelastung bei einer 5-Euro-Schwelle $21,7 \%$ betragen. Sie würde für die 6-Euro-Schwelle auf $26,1 \%$ steigen und für die 7-Euro-Schwelle auf 30,4 \%.

Im Hinblick auf die Mietkostenbelastung ist die $70 \%$-Schwelle somit von einer sozialpolitischen Perspektive aus betrachtet eine sehr ambitionierte wohnungspolitische Zielsetzung, die in vielen unteren Einkommensgruppen die Mietkostenbelastung unter $20 \%$ absenken würde, aber auch für 1-Personen-Haushalte eine Mietkostenbelastung unter 25 \% gewährleisten würde. Von dieser Zielsetzung her entspricht die 5-Euro-Schwelle der $70 \%$-Schwelle. Ein weniger ehrgeiziges, aber unter Umständen noch vertretbares sozialpolitisches Ziel wäre die 6-Euro-Schwelle. Diese Schwelle würde Mietkostenbelastungen um die $25 \%$ induzieren. Würde man $30 \%$ als noch akzeptabel betrachten, würde sich eine 7-Euro-Schwelle ergeben. 
Abbildung 1: Mietkostenbelastungen für unterschiedliche Schwellenwerte

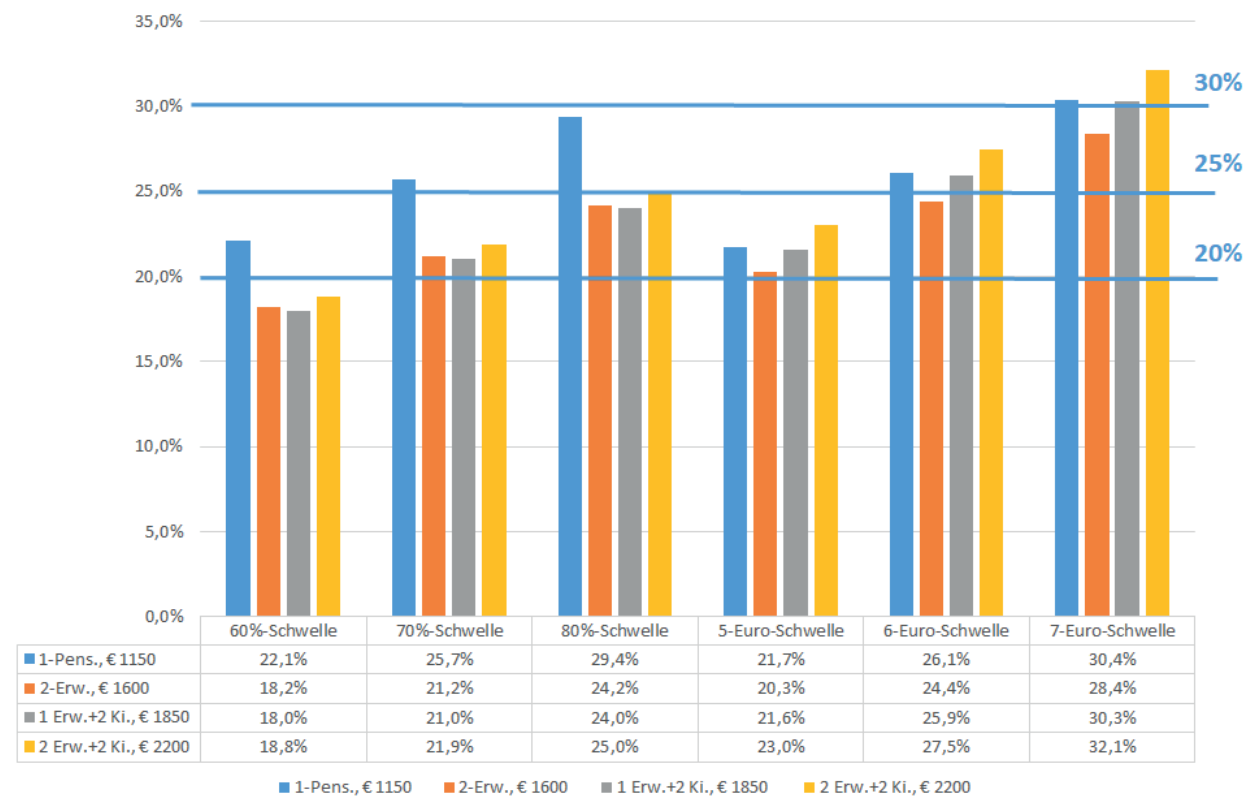

Quelle: MZ2017, eigene Berechnungen.

\subsection{Methodisches Vorgehen}

Zur Ermittlung von Signifikanzen und Vertrauensintervallen wurde der Designeffekt des MZ-Stichprobenplans berücksichtigt (Bacher und Tamesberger 2011; Bacher 2009), der dadurch entsteht, dass das MZDesign von einer einfachen Zufallsauswahl abweicht (Statistik Austria 2018e). Im Unterschied zu einer einfachen Zufallsauswahl arbeitet der MZ mit ungleichen Auswahlwahrscheinlichkeiten. Zudem bleibt ein einmal ausgewählter Haushalt fünfmal in der Stichprobe, was zu einem Klumpeneffekt und als Folge zu einem Genauigkeitsverlust führt. Zur Berechnung des Designeffekts wurde die von Ganninger (2006) vorgeschlagene Methode eingesetzt (Bacher und Tamesberger 2011). Sie resultiert in einen Designeffekt von 3,50, dem durch eine entsprechende Gewichtung der Fälle mit „1/DEFF“ Rechnung getragen wurde.

Berichtet werden durchgehend auf die jeweilige Population hochgerechnete Zahlenwerte.

Darüber hinaus werden Pfadanalysen (Holm 1977) durchgeführt. Bei der Pfadanalyse werden standardisierte Pfadkoeffizienten mit einem Absolutbetrag größer/gleich o,10 als bedeutsam betrachtet. Ziel der Pfadanalyse war, Zusammenhänge aufzudecken. Die Anordnung der Variablen (siehe Abbildungen 2 und 3) erfolgte dabei aus theoretischen Überlegungen (siehe Kapitel 2) zur Beantwortung der Forschungsfragen. Als letzte zu erklärende Variable wurde daher in den Pfadmodellen die Wahrscheinlichkeit betrachtet, dass eine günstige Wohnung in den letzten drei Jahren vermietet wurde. Das Modell enthält neben vermuteten kausalen Beziehungen auch logische Beziehungen, dass z. B. eine günstige Vermietung voraussetzt, dass es sich um eine Hauptmietwohnung handelt und nicht um eine Eigentumswohnung. Zudem ist eine durchgehende kausale Interpretation aller Zusammenhänge nicht möglich. So z. B. wird angenommen, dass eine Substandardwohnung häufiger günstig angeboten wird, dass also der Substandard die Ursache der günstigen Vermietung ist. Die Kausalität könnte auch in die andere Richtung gehen: Eine Wohnung wird deshalb nicht saniert, weil sie günstig vermietet ist. Trotz dieser Einschränkungen vermittelt die angewendete Pfadanalyse einen aufschlussreichen Einblick in die Bestimmungsfaktoren der untersuchten abhängigen Variablen, der sich zur Ableitung möglicher Maßnahmen eignet (siehe Abschnitt 5).

\section{Ergebnisse}

4.1 Anteil und Anzahl günstiger Mietwohnun-
gen in Österreich

Österreichweit stehen ca. 398.00o günstige Wohnungen in Hauptmiete zur Verfügung, also eine durchaus beachtenswerte Zahl. Dieser Wert liegt etwas höher als die von Statistik Austria berechnete Zahl von 373.000 Haushalten mit einer Wohnkostenbelastung (inkl. Energiekosten) über $40 \%$ (Statistik Austria 2018g). 
Tabelle 3: Anteil und Anzahl günstiger Wohnungen in Österreich 2017

\begin{tabular}{|c|c|c|c|}
\hline & Wert & $\begin{array}{l}\text { Unterer Wert des } \\
95 \% \text {-Vertrauensintervalls }\end{array}$ & $\begin{array}{c}\text { Oberer Wert des } \\
95 \% \text {-Vertrauensintervalls }\end{array}$ \\
\hline Bewohnte Wohnungen / Eigenheime & 3.889 .985 & & \\
\hline ... davon günstig (GÜNSTIG70) in \% & $10,23 \%$ & $9,84 \%$ & $10,62 \%$ \\
\hline Bewohnte Hauptmietwohnungen & 1.638 .548 & & \\
\hline ... davon günstig (GÜNSTIG70) in \% & $24,28 \%$ & $23,43 \%$ & $25,13 \%$ \\
\hline Anzahl günstiger Mietwohnungen & 397.945 & 382.697 & 413.194 \\
\hline $\begin{array}{l}\text {... davon günstig und in den letzten } 3 \text { Jahren vermietet } \\
\text { (GÜNSTIG703) in \% }\end{array}$ & $1,81 \%$ & $1,64 \%$ & $1,98 \%$ \\
\hline $\begin{array}{l}\text { Anzahl günstiger, in den letzten } 3 \text { Jahren vermieteter } \\
\text { Mietwohnungen }\end{array}$ & 70.409 & 63.699 & 77.118 \\
\hline
\end{tabular}

Quelle: MZ2017, eigene Berechnungen.

Lesehilfe: Im Jahr 2017 werden in Österreich 1.638548 Hauptmietwohnungen bewohnt. Davon sind 24,28 \% günstig vermietet. Der Gesamtwohnungsbestand inklusive Eigenheimen, Eigentumswohnungen und anderen Rechtsformen beträgt 3.889.985. Vom Gesamtbestand werden 10,23 \% günstig in Hauptmiete vermietet. In den letzten drei Jahren wurden 70.409 Hauptmietwohnungen günstig vermietet. Das sind $1,81 \%$ des Gesamtbestandes.

Tabelle 4: Anzahl günstiger Wohnungen in Hauptmiete für unterschiedliche Operationalisierung

\begin{tabular}{lllllll}
\hline & & $\mathbf{6 0} \%$-Schwelle & $\mathbf{7 0} \%$-Schwelle & 75 \%-Schwelle & 80 \%-Schwelle & 5-Euro-Schwelle \\
\hline Schwellenwert & In $€$ & 303,44 & 354,01 & 379,3 & 404,59 & 303,30 \\
Österreich & Absolut & 246.962 & 397.831 & 487.398 & 577627 & 261951 \\
& Index & 62,1 & 100,0 & 122,5 & 145,2 & 65,8 \\
\hline
\end{tabular}

Quelle: MZ2017, eigene Berechnungen.

Lesehilfe: Die $60 \%$-Schwelle bedeutet, dass die maximale Miete in einer günstigen Hauptmietwohnung $60 \%$ der Durchschnittsmiete einer Hauptmietwohnung mit gleicher Zimmerzahl betragen würde. Die entsprechende Mietobergrenze wäre € 303,44. Die Anzahl der günstigen Wohnungen in Hauptmiete würde sich von 397.831 (70 \%-Schwelle) auf 246.962 reduzieren. In Prozent ausgedrückt, wären dies nur 62,1 \% der Zahl an günstigen Wohnungen der $70 \%$-Schwelle.

Rein theoretisch würde somit in Bezug auf die Zahl der Haushalte mit einer hohen Wohnkostenbelastung eine ausreichend große Zahl an günstigen Wohnungen vorliegen. Allerdings steht dieser Rechnung die Tatsache gegenüber, dass sich räumliches Angebot und räumliche Nachfrage nicht decken müssen (z. B. günstige Wohnungen in Abwanderungsgebieten und Nachfrage in urbanen Räumen) und Personen, die nicht (mehr) von hoher Wohnkostenbelastung betroffen sind, in einer günstigen Wohnung leben. Bezogen auf die Zahl von ca. 3,9 Millionen Wohnungen (inkl. Eigenheimen) sind dies 10,2 \% (siehe Tabelle 3). Berücksichtigt man nur die Hauptmietwohnungen, so können 24,3 \% als günstig (leistbar) betrachtet werden, etwa jede vierte Hauptmietwohnung erfüllt das Kriterium "günstig“. Ein etwa im Vergleich zu $16 \%$ im Kanton Luzern (LUSTAT Statistik Luzern 2018) hoher Wert.

In den letzten Jahren wurden allerdings nur ca. 70.400 günstige Wohnungen in Hauptmiete vermietet. Bezogen auf den Gesamtbestand an allen Wohnungen (Miete und Eigentum) und Eigenheimen mit 1,8 \% ein vergleichsweise kleiner Wert.
Setzt man die Zahl der in den letzten drei Jahren vermieteten günstigen Wohnungen in Hauptmiete zur Gesamtzahl von ca. 600.000 an Neuvermietungen in den letzten drei Jahren in Beziehung, ergibt sich ein Anteil von 11,7 \%. Im Vergleich zu den $24,3 \%$ ist dies ein deutlicher Rückgang, der sich u.a. dadurch erklären lässt, dass günstige Wohnungen seltener auf den Markt kommen und Neubauwohnungen teurer vermietet werden. In Tabelle 4 wurden Vergleichsrechnungen für unterschiedliche Schwellenwerte vorgenommen. Eine Erhöhung von $70 \%$ auf $75 \%$ bei der Definition von günstigen Wohnungen würde den Bestand an günstigen Wohnungen in Hauptmiete in Österreich um 22,5 \% erhöhen, also von ca. 398.00o auf ca. 488.ooo.

Bivariate Analysen zeigen (Ergebnisse hier nicht angeführt), dass das Angebot von günstigen Wohnungen, wie aufgrund der Literatur zu erwarten, vom Errichtungsjahr der Wohnung, ihrer Ausstattung, dem Vermieter (Gemeinde, Genossenschaft, privat), der Wohnortgröße bzw. dem Bundesland und dem Mietverhältnis (befristet oder unbefristet) abhängt. Die Wahrscheinlichkeit, dass eine Wohnung günstig in 
Abbildung 2: Ergebnisse der explorativen Pfadanalyse für Österreich

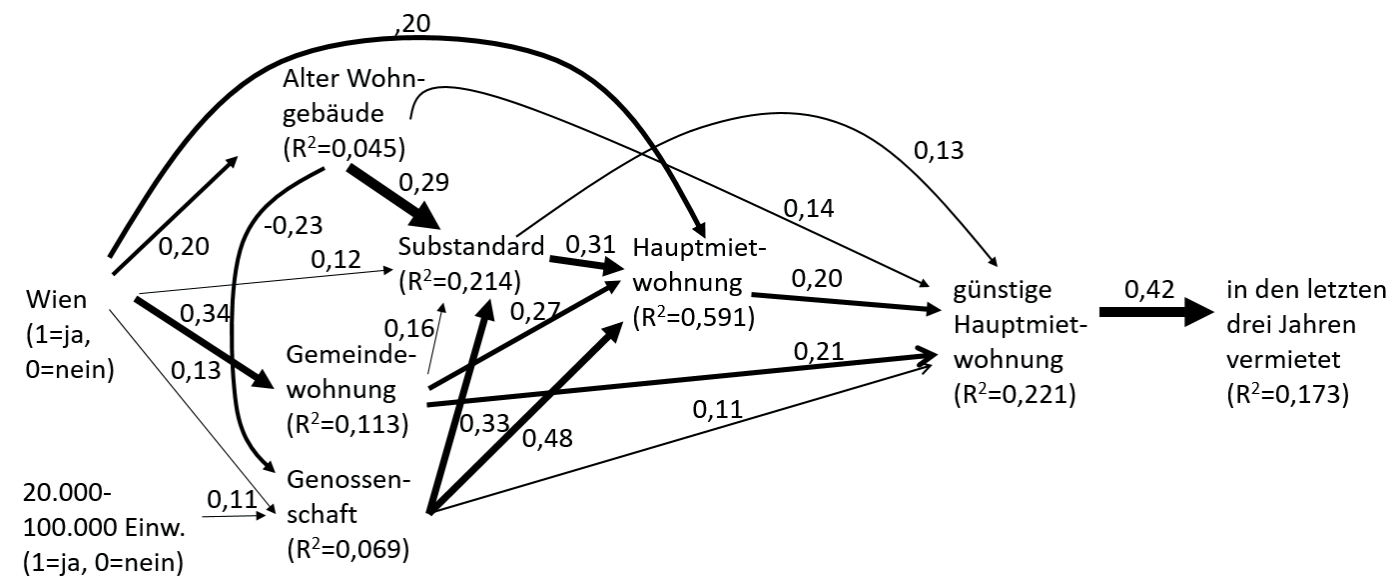

Quelle: MZ2017, eigene Berechnungen.

Anmerkungen: Ergebnisse einer explorativen Pfadanalyse. Nur standardisierte Pfadkoeffizienten mit einem Absolutbetrag $\geq 0,10$ eingezeichnet. Dicke der Pfeile drückt die Stärke des Zusammenhangs aus. Alle Koeffizienten mit p < o,oo1 signifikant. Multikollinearitätsdiagnostik mittels Varianz-Inflationsfaktor (VIF). VIF für alle Modelle kleiner 2,5 (maximaler VIF-Wert in allen Analysen = 2,495).

Hauptmiete angeboten wird, ist größer bei älteren Wohnungen, bei geringerer Ausstattung, wenn Gemeinden oder Genossenschaften die Wohnung vermieten und wenn das Mietverhältnis unbefristet ist. Bezüglich der Wohnortgröße nimmt der Anteil mit der Wohnortgröße zu (höhere Anteile in größeren Städten), wobei der Anteil in mittleren Städten mit einer EinwohnerInnenzahl zwischen 20.001 und 100.000 größer ausfällt als in Städten zwischen 100.000 und 300.000. Den höchsten Anteil an günstigen Wohnungen hat Wien mit einem Anteil von 20,9 \%.

\subsection{Einflussfaktoren auf günstige Mietwohnun- gen}

Mit Hilfe einer explorativen Pfadanalyse wurde untersucht, welche direkten und indirekten Zusammenhänge bestehen. Dabei wurde von einem einfachen Modell ausgegangen, das annimmt, dass die Zahl der in den letzten drei Jahren günstig vermieteten Hauptmietwohnungen von der Zahl der günstig vermieteten Hauptmietwohnungen abhängt, dass also ein bestimmter Prozentsatz von günstigen Hauptmietwohnungen in den letzten drei Jahren vermietet wurde. Für die Zahl der günstig vermieteten Hauptmietwohnungen wurde analog angenommen, dass sie von der Zahl der Hauptmietwohnungen abhängt und diese wiederum von dem Gesamtbestand an allen Wohnungen und Eigenheimen. Als Einflussfaktoren auf diese Bestandsgrößen wurden das Bundesland, der Vermieter, das
Alter des Wohngebäudes und der Wohnungsstandard spezifiziert (siehe Abbildung 2).

Geht man zunächst der Frage nach, von welchen Faktoren die Wahrscheinlichkeit abhängt, dass ein Wohnraum (Wohnung oder Eigenheim) als Hauptmietwohnung angeboten wird, so zeigen sich Abhängigkeiten vom Vermieter, vom Wohnungsstandard und vom Bundesland Wien ${ }^{6}$ (siehe Abbildung 2). Gemeinden und Genossenschaften als Vermieter bieten mit einer höheren Wahrscheinlichkeit Hauptmietwohnungen als andere Eigentümer an (Pfadkoeffizienten $\beta=0,27$ bzw. $ß=0,48)$. Als Hauptmietwohnungen werden zudem häufiger Wohnungen mit geringerem Wohnstandard $(ß=0,31)$ angeboten. Zudem zeigt sich die Sonderstellung von Wien. Selbst wenn angenommen wird - wie dies bei der Pfadanalyse der Fall ist -, dass sich Wien nicht in der Vermieterstruktur, im Alter des Wohnraums und in der Wohnausstattung unterscheidet, werden in Wien - ceteris paribus - mehr Wohnungen als Hauptmietwohnung angeboten $(\beta=0,20)$.

Liest man die Ergebnisse der Pfadanalyse (Abbildung 2) weiter nach rechts, so ist ersichtlich, dass die

6 Während bivariat das Bundesland einen signifikanten Einfluss hat, bleibt dieser Einfluss multivariat nur mehr für Wien bestehen. Andere dummy-Variablen wie z. B. für Westösterreich waren nicht signifikant.

7 Nach Allison (2012) gibt ein VIF-Wert kleiner 2,5 keinen Hinweis auf das Vorliegen von Multikollinearitätsprobleme und ermöglicht die Identifikation von Einflussfaktoren. 


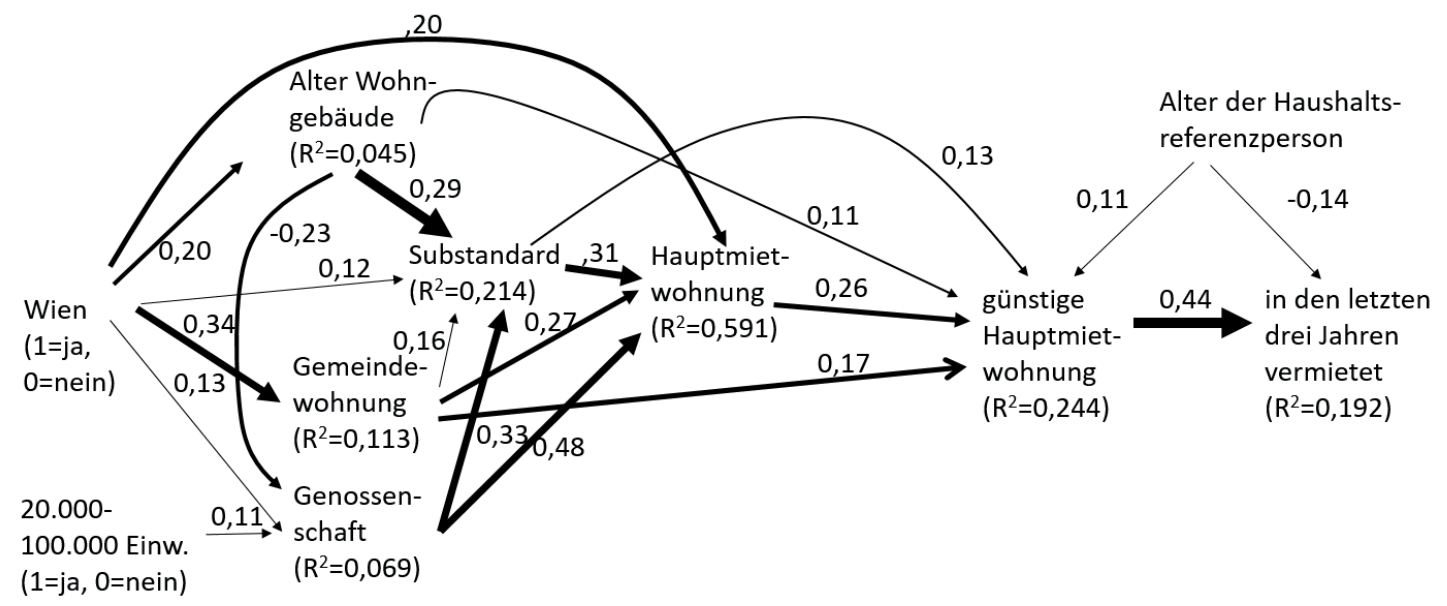

Quelle: MZ2017, eigene Berechnungen.

Anmerkungen: Ergebnisse einer explorativen Pfadanalyse. Nur standardisierte Pfadkoeffizienten mit einem Absolutbetrag $\geq 0,10$ eingezeichnet. Dicke der Pfeile drückt die Stärke des Zusammenhangs aus. Alle Koeffizienten mit p < o,oo1 signifikant. Multikollinearitätsdiagnostik mittels Varianz-Inflationsfaktor (VIF). VIF für zwei Modelle knapp über 2,5. ${ }^{8}$

Wahrscheinlichkeit, dass eine Hauptmietwohnung günstig angeboten wird, von fünf Faktoren abhängt. Voraussetzung ist zunächst, dass es sich um eine Hauptmietwohnung handelt. Dies bringt der Pfeil von der Hauptmietwohnung zur günstigen Hauptmietwohnung zum Ausdruck $(ß=0,20)$. Daneben hängt die Wahrscheinlichkeit, dass eine Wohnung als günstige Hauptmiete angeboten wird, vom Alter des Wohngebäudes, vom Wohnungsstandard und vom Vermieter ab. Günstig vermietete Wohnungen sind häufiger ältere Wohnungen $(\beta=0,14)$, haben einen geringeren Wohnstandard $(\beta=0,13)$, befinden sich häufiger im Besitz von Gemeinden ( $(=0,21)$ oder einer Genossenschaft $(\beta=0,11)$. Schließlich zeigt sich, dass die Zahl der in den letzten drei Jahren neu vermieteten günstigen Wohnungen ausschließlich vom Bestand an günstigen Wohnungen abhängt.

Das Pfaddiagramm eignet sich für die Erklärung der Sonderstellung von Wien und dem größeren Angebot an günstigen Wohnungen in Gemeinden mit 20.000 bis 100.000 EinwohnerInnen. Die Sonderstellung von Wien entsteht dadurch, dass Wien einen älteren Wohnungsbestand hat, es in Wien mehr

8 In den Analysen mit den abhängigen Variablen „günstige Hauptmietwohnung“ und „in den letzten drei Jahren ver-mietete günstige Hauptmietwohnung" überschreiten die VIF mit Werten von 2,738 und 2,830 knapp den Schwel-lenwert von 2,5 (Allison 2012). Die minimale Überschreitung kann unserer Ansicht nach toleriert werden, zumal entsprechend den Analysen von De Jongh et al. (2015) dieser Schwellenwert für große Datensätze zu restriktiv ist.
Gemeinde- und Genossenschaftswohnungen gibt und mehr Substandardwohnungen verfügbar sind. Diese Faktoren führen dazu, dass der Anteil an Hauptmietwohnungen insgesamt höher ist und dass auch mehr günstige Wohnungen zur Verfügung stehen, da die Wahrscheinlichkeit einer günstigen Hauptmietwohnung vom Anteil an Mietwohnungen am Gesamtbestand (in Wien höher), von der Vermieterstruktur (höherer Anteil bei Gemeindewohnungen und Genossenschaftswohnungen), dem Alter (mehr Angebot bei älteren Wohnungen) und dem Wohnungsstandard (mehr Angebot bei geringerem Wohnungsstandard) abhängt.

Im nächsten Analyseschritt schließlich wurden die Pfadanalysen um soziodemografische Variablen erweitert. ${ }^{9}$ Für Gesamtösterreich zeigt sich dabei (siehe Abbildung 3), dass in günstigen Wohnungen die Haushaltsreferenzpersonen (früher: Haushaltsvorstand) älter sind $\left(\aleph_{=0,11)}\right.$. Das Bild ändert sich, wenn zu den Vermietungen von günstigen Wohnungen in den letzten drei Jahren übergegangen wird. Es zeigt sich, dass die Neuvermietungen an jüngere Personen $(B=-0,14)$ gingen.

9 Die Erweiterung erfolgte dahingehend, dass angenommen wurde, dass die soziodemografischen Variablen einen Einfluss auf die beiden abhängigen Variablen „günstige Wohnungen“ und „in den letzten drei Jahren vermietet“ haben. Als sozio-demografische Merkmale wurden in die Analysen folgende Angaben zur Haushaltsreferenzperson einbezogen: Alter, Geschlecht, Bildung, Migrationshintergrund. 
Insgesamt kommt den soziodemografischen Merkmalen kein bedeutsamer Einfluss zu. Für das Geschlecht, die Haushaltsgröße, die abgeschlossene Bildung, den Migrationshintergrund usw. konnte kein bedeutsamer Pfadkoeffizient mit einem Absolutbetrag von größer/gleich o,10 ermittelt werden. Das ist u. a. dadurch begründet, dass der Einfluss der anderen Variablen, wie z. B. des Wohnungsangebots, statistisch kontrolliert wird. Interessant ist, dass der Bildung des Haushaltsvorstandes keine Wirkung zukommt. Eine derartige Wirkung hätte man erwarten können, da die Bildung mit dem Einkommen und damit mit der ökonomischen Situation der Haushalte korreliert. Man hätte daher erwarten können, dass Personen mit geringerer Bildung häufiger in günstigen Wohnungen leben.

Warum außer dem Alter keine soziodemografische Variable Wirkung zeigt, hat vermutlich mehrere Gründe. Der Befund kann zunächst als Hinweis darauf interpretiert werden, dass das Angebot an günstigem Wohnraum stark von der konkreten Wohnbaupolitik abhängt und für diese die aktuelle Soziodemografie nur eine untergeordnete Rolle spielt bzw. spielen kann. Ein weiterer Grund ist darin zu suchen, dass sich die Vergabe nicht an einem einzigen soziodemografischen Merkmal orientiert, sondern an einer Kombination dieser Merkmale. Als weiterer Grund kann hinzukommen, dass die Vermieter unterschiedliche Kombinationen dieser Merkmale verwenden. Schließlich ist zu beachten, dass sich das Modell auf ganz Österreich und - abgesehen von den Neuvermietungen von günstigen Wohnungen in den letzten drei Jahren - auf einen längeren Zeitraum bezieht. Zusammenhänge, die beispielsweise nur in einem Bundesland oder für einen bestimmten Zeitraum bestehen, können damit nicht aufgedeckt werden. Zusammenfassend zeigen die Einflussfaktoren in Abbildung 2 und 3, dass günstiger Wohnraum primär vom Angebot (an Hauptmietwohnungen, an älterem Wohnungsbestand, an Gemeinde- und Genossenschaftswohnungen, an Substandardwohnungen) abhängt und kaum von der Nachfrage (soziodemografische Merkmale).

\section{Zusammenfassung und Schlussfolgerungen}

Der vorliegende Beitrag hat untersucht, wie günstiger Wohnraum definiert werden kann, wie hoch die Anzahl an günstigen Mietwohnungen in Österreich im Jahr 2017 war und welche Einflussfaktoren es auf günstigen Wohnraum gibt. Als günstig wurden dabei in Anlehnung an das Konzept des Kantons Luzern
(LUSTAT Statistik Luzern 2018) Wohnungen mit einer Hauptmiete (inklusive Betriebskosten) bezeichnet, die weniger als $70 \%$ der durchschnittlichen Miete einer hinsichtlich der Raumzahl vergleichbaren Wohnung beträgt.

In Abhängigkeit von der Raumzahl ergeben sich folgende Schwellenwerte für günstige Hauptmietwohnungen im Jahr 2017:

- 1-Zimmer-Wohnung: € 234,82 bzw. € 6,06 pro $\mathrm{m}^{2}$ - 2-Zimmer-Wohnung: € 295,90 bzw. € 5,65 pro m² - 3-Zimmer-Wohnung: € 339,40 bzw. € 4,94 pro m² - 4-Zimmer-Wohnung: $€ 388,89$ bzw. $€ 4,57$ pro m² - 5-Zimmer-Wohnung: € 481,93 bzw. € 4,29 pro m²

- 6 und mehr Zimmer: $€ 654,38$ bzw. $€ 4,10$ pro m².

Entsprechend der Schwellenwerte wird eine 1-Zimmer-Wohnung dann als günstig bezeichnet, wenn die Hauptmiete inkl. Betriebskosten weniger als $€ 234,82$ ausmacht. Die durchschnittlichen Mieten pro Quadratmeter variieren abhängig von der Raumzahl zwischen $€ 6,06$ und $€ 4,10$. Über alle Wohnungsgrößen hinweg ergibt sich ein durchschnittlicher Schwellenwert von $€$ 5,01 pro $\mathrm{m}^{2}$, der dem 5-Euro-Wohnmodell des Landes Tirol für günstige Wohnungen entspricht.

In Bezug auf die ausgewählten Haushalte mit einem Nettoeinkommen knapp über der Einkommensgrenze der Wohnbeihilfe Oberösterreich resultieren Mietkostenbelastungen von 21,0 \% bis $25,7 \%$. Die Belastungen liegen zumeist unter der nationalen Schwelle von 25,0 \% für eine Mietkostenbelastung. Von einem sozialpolitischen Standpunkt aus betrachtet stellt die $70 \%$-Schwelle somit eine sehr ambitionierte Zielsetzung dar.

Die mit dem Schwellenwert von 70 \% ermittelte Zahl an günstigen Hauptmietwohnungen beträgt 398.000 und ist also durchaus beachtenswert. Allerdings wurden von diesen Wohnungen nur 70.400 in den letzten drei Jahren vermietet.

Als bedeutsame Faktoren für das Angebot an günstigem Wohnraum in Hauptmiete erwiesen sich das Bundesland Wien, die Einwohnerzahl, das Errichtungsjahr, der Wohnstandard, der Vermieter und das Mietverhältnis. Wenig überraschend sind günstige Wohnungen älter, haben einen geringeren Wohnstandard und sind häufiger im Besitz einer Gemeinde oder Genossenschaft. Es liegt - sofern die Wohnung länger bewohnt wird - auch häufiger ein unbefristetes Mietverhältnis vor. Mittels explorativer Pfadanalysen wurde analysiert, welches Gewicht den einzelnen Faktoren zukommt. Im Wesentlichen wurde dabei ein einfaches Wohnungsangebotsmodell bestätigt, wonach die 
Abbildung 4: Ergebnisse der explorativen Pfadanalyse für Österreich

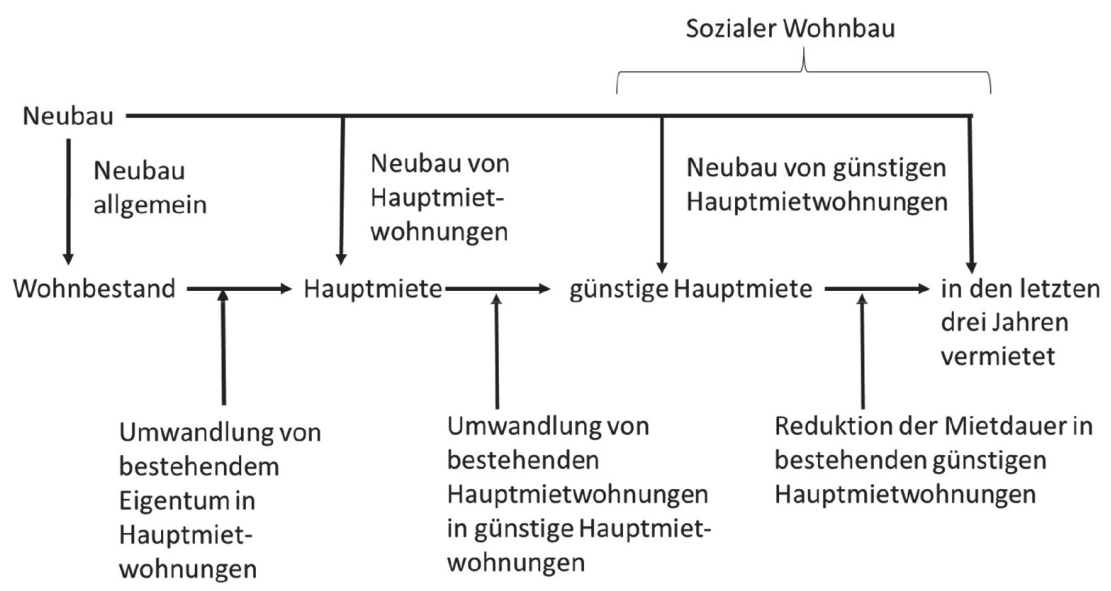

Quelle: eigene Darstellung

Wahrscheinlichkeit, dass eine günstige Hauptmietwohnung in den letzten drei Jahren vermietet wurde, vom Bestand an günstigen Hauptmietwohnungen abhängt, der wiederum davon abhängt, wie viele Hauptmietwohnungen verfügbar sind. Dabei kommt für das günstige Angebot Genossenschaften und Gemeinden (primär in Wien) als Vermieter eine besondere Bedeutung zu. Die Anzahl von Hauptmietwohnungen hängt wiederum vom Verhältnis von Eigentums- zu Mietwohnungen ab.

Ziel des Beitrags war die Entwicklung eines Indikators für günstige Wohnungen für ein systematisches Monitoring, wozu der Mikrozensus genutzt werden sollte. Der Mikrozensus als Datenbasis erwies sich für viele Fragestellungen als geeignet. Der untersuchte Indikator (70 \%-Schwelle) oder Modifikationen von ihm (z. B. 75 \%-Schwelle oder Festlegung eines absoluten Schwellenwertes) stellen unseres Erachtens eine sinnvolle Ergänzung zu den verfügbaren Daten zum Wohnungsmarkt dar und tragen der Tatsache Rechnung, dass der Wohnungsmarkt stark vom Angebot abhängt. Die 70 \%-Schwelle oder ähnliche Indikatoren bilden somit das Angebot an günstigen Wohnungen ab.

Statistisch betrachtet besitzen die auf diese Weise berechneten Indikatoren eine relativ hohe Sensitivität. D. h. eine geringfügige Änderung der Schwelle kann zu einer deutlichen Reduktion oder Erhöhung der Zahl der günstigen Hauptmietwohnungen führen. Daher ist eine sorgfältige, gut begründete und letztlich politisch zu begründende Auswahl des Schwellenwertes notwendig. Dabei sind u. a. folgende Fragen zu beantworten:

- Soll ein absoluter oder ein relativer Wert verwendet werden?

- Was ist die Bezugsgröße? Denkbare Bezugsgrößen wären z. B. die durchschnittliche Miete pro m2 oder die Durchschnittsmiete nach Anzahl der Zimmer. Alternativ zum Durchschnitt könnte der Median als Bezugsgröße herangezogen werden.

- Soll der Index für Österreich insgesamt berechnet werden oder getrennt für bestimmte Teilgruppen/ -aggregate, wie z. B. Bundesländer oder Städte?

- Sollen weitere Zielvorgaben berücksichtigt werden? Neben den hier im Vordergrund stehenden sozialpolitischen Aspekten könnten andere zu beachtende Zielvorgaben sein: faire Arbeitsbedingungen am Bau, angemessener Wohnstandard, Barrierefreiheit, altersgerechte und lebensphasen-orientierte Bauformen, geringer Energieverbrauch usw.

Grundsätzlich wäre es möglich, den Index für Teilaggregate, z. B. Bundesländer oder bestimmte Gemeindegrößen, zu berechnen. Allerdings können sich dabei sehr rasch Beschränkungen wegen kleiner Fallzahlen ergeben. Sollen zusätzliche Zielvorgaben berücksichtigt werden, sind Zusatzerhebungen erforderlich. Wünschenswert wäre auf jeden Fall eine Erfassung des Leerstandes und der Wohnmarktdynamik.

Die durchgeführten Pfadanalysen geben Anhaltspunkte für mögliche Maßnahmen zur Erhöhung der Zahl der günstigen Wohnungen. Ihre Ergebnisse sind auch relativ robust gegenüber der Wahl des Schwellenwertes. Aus ihnen lassen sich die in Abbildung 4 angeführten Maßnahmen ableiten, wobei jene Maßnahmen am stärksten wirken, die unmittelbar auf die entsprechende Zielgröße Einfluss haben. Soll z. B. die Zahl der in drei Jahren vermieteten günstigen Wohnungen erhöht werden, so geht die stärkste Wirkung von Neubauten von günstigen Wohnungen sowie von einer Reduktion der Mietdauer in günstigen Wohnungen aus. Die Ankurbelung des Wohnbaus allgemein hätte 
dagegen eine schwächere Wirkung, da sie nur indirekt wirkt. Durch sie wird der Wohnbestand erhöht. Ein erhöhter Wohnbestand würde in der Folge auch zu mehr Hauptmietwohnungen führen, mehr Hauptmietwohnungen auch zu mehr günstigen Mietwohnungen usw. Allerdings würde dabei ein Filtereffekt eintreten, da nicht alle Neubauten Hauptmietwohnungen wären (Filter: Mietanteil unter Neubauten) und nicht alle neugebauten Hauptmietwohnungen günstig vermietet werden (Filter: Anteil günstiger Hauptmietwohnungen an allen Hauptmietwohnungen).

Damit durch Neubauten mehr günstiger Wohnraum initiiert wird, wäre es notwendig, den Anteil von Hauptmietwohnungen und - noch wirksamer - den Anteil günstiger Hauptmietwohnungen bei Neubauten zu erhöhen. Denkbare Maßnahmen wären:

- Förderung der Errichtung von Mietwohnungen anstelle von Wohnungs-/Hauseigentum.

- Stärkere Konzentration von Wohnbauförderungen auf Gemeinden und Genossenschaften, da diese mit einer größeren Wahrscheinlichkeit (günstige) Mietwohnungen errichten.

- Neubauten in Gemeinden mit günstigen Bodenpreisen, wobei gleichzeitig eine gute öffentliche Verkehrsanbindung zu den Ballungsräumen und Arbeitsstätten gewährleistet werden müsste.

- Verdichteter Hochhausbau in Städten zur Amortisation hoher Grundpreise. Durch Hochhäuser würde sich ein geringerer Quadratmeterpreis pro Wohnung ergeben, der aber möglicherweise durch höhere Baukosten bei Hochhäusern kompensiert wird.

- Reduktion des Wohnstandards. Dies würde aber zu einer sichtbaren „Zwei-Klassen-Struktur“ führen und zu einer stärkeren Segregation mit allen damit verbundenen Problemen.

- Quotenregelung bei Baulandwidmungen. Bei der Umwidmung in Bauland und dem anschließenden Verkauf verpflichten sich die ErwerberInnen, einen bestimmten Prozentsatz für günstige Wohnungen zur Verfügung zu stellen. ${ }^{10}$

- Quotenregelung bei Wohnbauförderung. Die BauträgerInnen verpflichten sich, einen bestimmten Prozentsatz der erbauten Wohnungen günstig zu vermieten, wenn sie eine öffentliche Förderung erhalten.

10 Diesen Weg hat die Stadt Wien mit einer Änderung der Flächenwidmung am 21. März 2019 beschlossen (siehe Stadt Wien 2019. Online: https://www.wien.gv.at/bauenwohnen/bauordnungsnovelle-gefoerderter-wohnbau.html [31.10.2019]).
Neben der Erhöhung von Neubauten von günstigen Hauptmietwohnungen würde eine Reduktion der Mietdauer in den günstigen Hauptmietwohnungen die Zahl der Neuvermietungen rasch erhöhen. D. h. es müsste regelmäßig geprüft werden, ob noch der Anspruch auf eine günstige Mietwohnung besteht. Diese Maßnahme hätte aber den Nachteil, dass es zu einer hohen Fluktuation käme und die soziale Durchmischung abnehmen würde.

Eine weitere Maßnahme, günstigen Wohnraum zu schaffen, wäre die Umwandlung von Hauptmietwohnungen in günstige Hauptmietwohnungen, etwa - wie derzeit politisch mitunter gefordert ${ }^{11}$ durch den Ankauf von älteren Wohnungen durch Gemeinden und Genossenschaften. Da es sich um ältere Wohnungen handelt, sind die Quadratmeterpreise vermutlich geringer und die Vermieter wären in der Lage, die Wohnungen in Hauptmiete günstig zu vermieten. Auch durch die Einführung von Mietobergrenzen für Neuvermietungen am privaten Wohnungsmarkt könnte das Angebot an günstigen Wohnungen erhöht werden (Tockner 2012). Regulative Eingriffe durch „Mietpreisbremsen“ oder durch einen sogenannten „Mietendeckel“ wie in Berlin, der alle Neuvermietungen (außer im Neubau) für die nächsten fünf Jahre einfrieren soll, haben kontroverse Diskussionen hervorgerufen (siehe z. B. Holm 2014; Michelsen und Mense 2018; Priewe 2019; Voigtländer 2019). Hintergrund der Kontroverse ist natürlich ein Interessenskonflikt, da ein "Mietendeckel“ - wenn dieser effektiv ist - die Renditen von Wohnungseigentümern, die vermieten, beschränkt bzw. verlangsamt zugunsten einer sozialen Wohnraumversorgung (Rink et al. 2015; Priewe 2019).

Denkbar wäre auch eine Quotenregelung bei geförderter Sanierung. Wird ein älteres Haus saniert, wird die Auflage gemacht, dass ein bestimmter Prozentsatz günstig in Hauptmiete angeboten werden muss. In eine andere Richtung gehen könnten Maßnahmen der Subjektförderung, bei der Personen unterstützt werden, damit sie sich eine Mietwohnung leisten oder Eigentum erwerben können. ${ }^{12}$

11 So z. B. plant die Stadt Berlin laut Medienberichten den Rückkauf von Wohnungen (siehe: Berliner Morgenpost vom 11.01.2019. Online: https://www.morgenpost.de/berlin/ article216184471/Mueller-will-privatisierte-GSW-Wohnungen-zurueckkaufen.html [29.01.2019]).

12 Eine weitere indirekte Maßnahme wäre, die Nettoeinkommen der Haushalte zu erhöhen, damit sie sich teurere Wohnungen leisten könnten. 
Zusammenfassend zeigen die hier vorgenommenen Überlegungen, dass eine Erhöhung der Zahl der zur Vermietung verfügbaren günstigen Wohnungen wünschenswert wäre. Unmittelbar wirksame Maßnahmen wären der Bau von günstigen Mietwohnungen, der Ankauf von bestehenden Mietwohnungen durch Gemeinden und Genossenschaften und deren anschließende günstige Vermietung sowie die Verkürzung der Mietdauer in günstigen Wohnungen. Eine Alternative zur Erhöhung der Zahl der verfügbaren günstigen Wohnungen wäre ein Ausbau der Subjektförderung. Insgesamt sind bei Maßnahmen (ungeplante) Nebenwirkungen und Nebenkosten zu beachten. Möglicherweise gelingt eine günstige Vermietung, allerdings um den Preis höherer Energie- und Mobilitätskosten, wenn die Wohnung nicht ausreichend saniert und vom Arbeitsort weiter entfernt ist.

\section{Literatur:}

Allison, P. (2012): When can you safely ignore multicollinearity? Statistical Horizons Blog. Online: https://statisticalhorizons.com/multicollinearity [31.10.2019].

Amt der Tiroler Landesregierung (Hg.) (2017): Leistbares Wohnen. Gemeinde Inzing plant 5-Euro-Wohnanlage.

Bacher, J. (2009): Analyse komplexer Stichproben. In: Weichbold, M./Bacher, J./Wolf, Ch. (Hg.): Umfrageforschung. Wiesbaden: VS Verlag für Sozialwissenschaften, 253-272.

Bacher, J./Tamesberger, D. (2011): Junge Menschen ohne (Berufs-)Ausbildung. WISO, 33 (4), 95-112.

Beer, C./Wagner, K. (2017): Household's housing expenditure in Austria, Germany and Italy. Monetary Policy and the Economy (Q4). Online: https://www.hfcs.at/publikationen/oenbpublikationen.html [25.05.2018].

Blaas, W./Wieser, R. (2004): Einfluss von Wohnbauförderung und Richtwertsystem auf die Mietenentwicklung. Wien: AK Wien.

De Jongh, PJ./de Jongh, E./Pienaar, M./ Gordon-Grant, H./M Oberholzer, M./L Santana, L. (2015): The impact of preselected variance inflation factor thresholds on the stability and predictive power of logistic regression models in credit scoring. Orion, 31 (1), 17-37.

Deschermeier, P./Haas, H./Voigtländer, M. (2015): Anbieter von günstigem Wohnraum - eine Analyse für zehn Großstädte in NRW in Kooperation mit der LEG Immobilien AG. IW policy paper, 35. Online: https://www.iwkoeln.de/studien/iw-policy-papers/beitrag/philipp-deschermeier-heide-haas-michael-voigtlaender-anbietervon-guenstigem-wohnraum-251397.html [24.05.2018].

Egner, B./Grabietz, K. (2017): In search of determinants for quoted housing rents: Empirical evidence from major German cities. Urban Research \& Practice, 11 (4), 460477.
Fehlberg, T./Mießner, M. (2015): Mietpreissteigerungen und Wohnungsengpässe abseits der Ballungsräume. Investitionen in Wohnimmobilien in B-Lagen und Regionalzentren - das Beispiel Göttingen. Zeitschrift für kritische Stadtforschung, 3 (1), 25-44.

Fritzsche, C./Kluge, J. (2014): Wodurch werden die Mietpreise bestimmt? Unterschiede in den Mieten in Ost- und Westdeutschland. Dresden: Ifo, 21 (6), 3-10.

Ganninger, M. (2006): Estimation of Design Effects for ESS Round II. Online: http://www.europeansocialsurvey.org/ docs/round2/methods/ESS2_estimation_of_design_effects.pdf [19.07.2019].

Girouard, N./Kennedy, M./Van den Noord, P./André, Ch. (2006): Recent House Price Developments: The Role of Fundamentals. OECD Working paper 475.

Holm, A. (2014): Feigenblatt Mietpreisbremse. Blätter für deutsche und internationale Politik, 5, 20-22.

Holm, A. (2018): Wohnen macht arm. WSI-Mitteilungen, 71 (5), 427-428.

Holm, K. (1977): Lineare multiple Regression und Pfadanalyse. In: Holm, K. (Hg.): Die Befragung, Bd. 5. München, 7-102.

Institut für Wohnen und Umwelt (IWU) (2005): Auswirkungen des Wegfalls von Sozialbindungen und des Verkaufs öffentlicher Wohnungsbestände auf die Wohnungsversorgung unterstützungsbedürftiger Haushalte. Teilbericht präsentiert im Rahmen des vom BMBF geförderten Forschungsverbundes „Wohnungslosigkeit und Hilfen in Wohnungsnotfällen". Online: https://www.iwu.de/ forschde/dateien/zwischenbericht_auswirkungen.pdf [02.11.2018].

Kunnert, A. (2016): Leistbarkeit von Wohnen in Österreich. Operationalisierung und demographische Komponenten. Wien: WIFO. Online: http://www.wifo.ac.at/wwa/pub$\mathrm{id} / 58932$ [11.06.2018].

Land OÖ (2018a): Wohnbau Bericht 2017. Leistungen - Finanzierung - Ziele. Online: www.land-oberoesterreich.gv.at [17.11.2018].

Land OÖ (2018b): Wohnbeihilfe. Online: https://www.landoberoesterreich.gv.at/wohnbeihilfe.htm [16.11.2018].

Lang, R./Stöger, H. (2018): The role of the local institutional context in understanding collaborative housing models: empirical evidence from Austria. International Journal of Housing Policy, 18 (1), 35-54.

LUSTAT Statistik Luzern (2018): Angebot an günstigem Wohnraum. Online: https://www.lustat.ch/indikatoren/ sozialindikatoren/wohnen/angebot-an-guenstigemwohnraum [24.05.2018].

Michelsen, C./Mense, A. (2018): Evaluierung der Mietpreisbremse. Endbericht. Berlin: DIW.

Möbert; J./Kortmann, K./ Nemeth, R. (2008): Hedonische Regression der Wohnungsmietpreise unter Berücksichtigung von Lagevariablen am Beispiel eines Bestands im Ruhrgebiet. Zeitschrift für Immobilienökonomie, (1), 50-64. 
Mundt, A./Amann, W. (2015): Leistbares Wohnen - Bestandsaufnahme von monetären Leistungen für untere Einkommensgruppen zur Deckung des Wohnbedarfs. Im Auftrag des Sozialministeriums. Online: www.iibw.at [31.05.2018].

Mundt, A. (2017): Housing benefits and minimum income schemes in Austria - an application of the residual income approach to housing affordability of welfare recipients. International Journal of Housing Policy, 4, 1-29.

OeNB (2018a): Immobilienmarktanalyse. Online: https:// www.oenb.at/Geldpolitik/schwerpunkt_immobilienmarktanalyse.html [25.05.2018].

OeNB (2018b): Immobilienpreisindex. Online: https://www. oenb.at/Statistik/Standardisierte-Tabellen/Preise-Wettbewerbsfaehigkeit/Sektorale-Preisentwicklung/immobilienpreisindex.html [24.11.2018].

Priewe, J. (2019): Warum der Berliner Mietendeckel ein Schritt in die richtige Richtung ist. Online: https://makronom. de/wohnungsmarkt-warum-der-berliner-mietendeckel-ein-schritt-in-die-richtige-richtung-ist-32182 [18.07.2019].

Rink, D./Schönig, B./Gardemin, D./Holm, A. (2015): Städte unter Druck. Die Rückkehr der Wohnungsfrage. Blätter für deutsche und internationale Politik, 6, 69-79.

Saiz, A. (2003): Room in the Kitchen for the Melting Pot: Immigration and Rental Prices. Review of Economics and Statistics, 85 (3), 502-521.

Saiz, A. (2007): Immigration and housing rents in American cities. Journal of Urban Economics, 61 (2), 345-371.

Schoibl, H./Stöger, H. (2014): Armutsfalle Wohnen. Wohnpolitik und Armutsrisiken. In: Dimmel, N./Schenk, M./ Stelzer-Orthofer, Ch. (Hg.): Handbuch Armut in Österreich. 2., voll. überarb. und erweit. Aufl. Innsbruck [u.a.]: StudienVerlag, 309-319.

Schönig, B. (2013): Die neue Wohnungsfrage. Blätter für deutsche und internationale Politik, 2 (2013), 17-20.

Springler, E. (2010): Finanzkrise und die steigende finanzielle Belastung der Haushalte durch Wohnkosten. Kurswechsel, 1 (2010), 68-76.

Springler, E. (2014): Lehren aus der Immobilienkrise. Einbettung des Wohnungsmarktes in eine theoretische postkeynesianische Analyse. Kurswechsel, 3 (2014), 40-47.

Statistik Austria (2018a): Baukostenindex für den Wohnhausund Siedlungsbau, Gesamtbaukosten ab Basisjahr 1990, 2000, 2005, 2010, 2015. Online: http://www.statistik.at/ web_de/statistiken/wirtschaft/preise/baukostenindex/ index.html [19.07.2019].

Statistik Austria (2018b): Baupreisindex. Wien: Statistik Austria. Online verfügbar unter: http://www.statistik.at/ web_de/statistiken/wirtschaft/preise/baupreisindex/index.html [19.07.2019].

Statistik Austria (2018c): Brutto- und Nettojahreseinkommen der unselbständig Erwerbstätigen 1997 bis 2017 Online: http://www.statistik.at/web_de/statistiken/menschen_ und_gesellschaft/soziales/personen-einkommen/jaehrliche_personen_einkommen/index.html [24.11.2018].
Statistik Austria (2018d): Jährliche Personeneinkommen. Online: http://www.statistik.at/web_de/statistiken/ menschen_und_gesellschaft/soziales/personen-einkommen/jaehrliche_personen_einkommen/index.html [07.10.2018].

Statistik Austria (2018e): Mikrozensus ab 2004. Online: https://www.statistik.at/web_de/frageboegen/private_ haushalte/mikrozensus/index.html [19.7.2019].

Statistik Austria (2018f): SILC. Online: http://www.statistik. at/web_de/frageboegen/private_haushalte/eu_silc/index.html [31.05.2018].

Statistik Austria (2018g): Tabellenband Wohnen 2017, Kapitel 2: EU-SILC. Online: http://www.statistik.at/web_de/ statistiken/menschen_und_gesellschaft/wohnen/wohnkosten/index.html [16.11.2018].

Statistik Austria (2018h): Wohnen. Online: http://www.statistik.at/web_de/statistiken/menschen_und_gesellschaft/ wohnen/index.html [23.05.2018].

Statistik Austria (2018i): Wohnen 2017 - Zahlen, Daten und Indikatoren der Wohnstatistik. Wien: Statistik Austria.

Statistik Austria (2019): Armutsgefährdungsschwelle 2018 bei $60 \%$ des Medians für unterschiedliche Haushaltstypen. Online http://www.statistik.at/web_de/statistiken/ menschen_und_gesellschaft/soziales/armut_und_soziale_eingliederung/index.html [30.07.2019].

Stöger, H. (2017): Wohnungsversorgung als öffentliche Verantwortung? Das Beispiel des sozialen Wohnungssektors. WISO, 40 (3), 99-117.

Tockner, L. (2012): Mietensteigerungen in Österreich und Wien. Auswertungen aus dem Mikrozensus. Online: https://wien.arbeiterkammer.at/service/studien/wohnen/ Mietensteigerungen_in_Wien_und_Oesterreich.html [29.07.2019].

Tockner, L. (2017): Wohnungsmieten und Wohnungspreise in Wien 2015. Wien: AK Wien.

Voigtländer, M. (2019): Wohnungsmarkt: Mietendeckel verschlimmert Lage. Wirtschaftsdienst, 7 (99), 448-449.

Wieser, R. (o.J.): Stabilisierende und destabilisierende Faktoren auf den Wohnungsmärkten der EU-14. Online: http://www.gbv.at/Document/View/4274 [18.07.2019].

Zucha, V. (2016): Sozialer Wohnbau bremst Mietpreisdynamik. Österreichische Gemeinde-Zeitung (ÖGZ), 9, 11-13. 